\title{
Plasma osteocalcin levels as a predictor of cardiovascular disease in older men and women: a population-based cohort study
}

\author{
Kristin Holvik ${ }^{1,2}$, Natasja M van Schoor ${ }^{2}$, Elisabeth M W Eekhoff ${ }^{3}$, \\ Martin den Heijer ${ }^{3}$, Dorly J H Deeg ${ }^{2}$, Paul Lips ${ }^{3}$ and Renate de Jongh ${ }^{3}$ \\ ${ }^{1}$ Division of Epidemiology, Norwegian Institute of Public Health, PO Box 4404 Nydalen, N-0403 Oslo, Norway, \\ ${ }^{2}$ Department of Epidemiology and Biostatistics, EMGO Institute for Health and Care Research and ${ }^{3}$ Endocrine \\ Section, Department of Internal Medicine, VU University Medical Center, Amsterdam, The Netherlands
}

Correspondence should be addressed to K Holvik Email kristin.holvik@fhi.no

\begin{abstract}
Objective: The role of osteocalcin (OC) in cardiovascular disease (CVD) is unresolved. We aimed to study the association between plasma OC concentrations and the risk of non-fatal and fatal CVDs. We also aimed to investigate whether such an association, if present, would be mediated by established metabolic risk factors.

Design: A population-based longitudinal cohort study.

Methods: In 1995/1996, OC was determined in blood samples drawn from 1319 subjects aged 65-88 years participating in the Longitudinal Aging Study Amsterdam in 1995/1996. The self-reported CVD events were collected every 3 years until 2005/2006, and CVD deaths until 1st January 2007. Cox proportional hazards regression was performed, considering potential confounders (smoking, physical activity, and BMI) and mediators (blood pressure, plasma triglycerides, total and HDL cholesterol, fructosamine, and aortic calcification).

Results: During the median 4.1 years follow-up, 709 subjects (53.8\%) suffered a CVD event. There was no overall association between OC and CVD: hazard ratio (HR) was 0.97 (95\% Cl 0.90-1.04) per nmol/l higher plasma OC, adjusted for age and sex. There was a statistical interaction between plasma OC, age, and sex on CVD $(P=0.014)$. In those subjects aged $\geq 75$ years, age-adjusted HRs $(95 \% \mathrm{Cl})$ were $0.86(0.75-0.99)$ in men and $1.16(1.03-1.31)$ in women per nmol// higher plasma OC. Adjustment for covariates only slightly attenuated the association in older-old men, but did not affect the association in older-old women.

Conclusion: A higher plasma OC concentration was associated with a reduced risk of CVD in older-old men and with an increased risk of CVD in older-old women. We found no evidence that this was mediated by arterial calcification or metabolic risk factors.
\end{abstract}

\section{Introduction}

Osteocalcin (OC (BGLAP)) is a non-collagenous protein expressed by the bone-forming osteoblasts (1), thought to be central in bone mineralization. Elevated OC levels have been shown to be associated with osteoporosis (2). OC is also expressed in calcified atherosclerotic lesions, where it is believed to be a mediator in the calcification process $(1,3,4,5)$. Vascular calcification appears to be an organized process corresponding to bone mineralization, regulated by several bone-associated proteins including OC.

However, bone cells also participate in glucose metabolism via OC secretion, and this may have an impact on the risk of cardiovascular disease (CVD) (6). In animal studies, OC seems to act on pancreatic $\beta$-cells and
(C) 2014 European Society of Endocrinology Printed in Great Britain
Published by Bioscientifica Ltd. 
adipocytes to regulate insulin secretion and sensitivity and fat mass $(7,8)$. Knockout mice lacking $O c$ showed decreased $\beta$-cell proliferation, glucose intolerance, and insulin resistance (7). This notion is supported by recent human studies, which have consistently reported inverse associations between circulating $\mathrm{OC}$ and components of the metabolic syndrome in community-dwelling populations $(9,10,11$, $12,13,14,15)$. A low plasma OC concentration was strongly associated with a higher prevalence of metabolic syndrome in the Longitudinal Aging Study Amsterdam (LASA) (16). This was mediated through an association with blood levels of triglycerides and HDL cholesterol, waist circumference, and hypertension.

The role of OC in CVD remains to be clarified. While some patient studies have demonstrated inverse associations between circulating $\mathrm{OC}$ and $\operatorname{CVD}(17,18,19)$, a large cohort study in community-dwelling older men aged 70-89 years found the highest CVD mortality at a high serum OC concentration (20).

We aimed to investigate whether plasma OC levels were independently associated with the risk of non-fatal and fatal CVDs during up to 11 years of follow-up. We also aimed to investigate whether such an association, if present, would be mediated by atherosclerosis as defined by aortic calcification scores (ACSs) or by cardiovascular risk factors including blood pressure, glucose tolerance status, and blood lipids.

\section{Subjects and methods}

Data were collected as part of LASA, an ongoing population-based cohort study in older people in The Netherlands. The cohort has been described in detail previously (21). Briefly, a sample of older men and women (aged 55-85 years at baseline), stratified by age, sex, urbanization grade, and expected 5-year mortality, was drawn from the population registers of 11 municipalities in three regions in The Netherlands, being a representative sample of the Dutch population. At baseline (1992/1993) and every third year thereafter, subjects participated in home interviews performed by trained nurses.

\section{Study subjects}

The current study included a subgroup participating in the second medical interview of LASA (1995/1996), aged $\geq 65$ years, and living in Amsterdam, Zwolle, and Oss and surroundings $(n=1509)$. After the medical interview at home, participants were invited to the VU University Medical Center (VUmc) or to hospitals in Zwolle (East) and Oss (South) or a health care center near their homes in the morning after a light (dairy-free) breakfast, where blood and urine samples were collected ( $n=1319$, constituting $87.4 \%)$. Participants were interviewed in three consecutive cycles (1998/1999, 2001/2002, and 2005/2006) about cardiovascular events that had occurred since their previous interview.

\section{Biochemical assays}

Urine and blood samples were centrifuged and stored at -20 or $-70{ }^{\circ} \mathrm{C}$ until determination in $1997 / 1998$ at the Endocrine Laboratory in VUmc.

Plasma intact OC was determined using an IRMA (Biosource Diagnostics, now DIAsource ImmunoAssays, Louvain-la-Neuve, Belgium) (22). The interassay coefficient of variation $(\mathrm{CV})$ values were $8 \%$ at $3.5 \mathrm{nmol} / \mathrm{l}$ and $5 \%$ at $0.7 \mathrm{nmol} / \mathrm{l}$.

Overnight urinary excretion of the bone resorption marker deoxypyridinoline (DPD) was determined by a competitive immunoassay on an automated ACS 180 System (Chiron Diagnostics, Emeryville, CA, USA). The values were corrected for creatinine $(\mathrm{Cr})$ concentration in the same urine sample. The interassay CV value was 5\% (23).

In 2005, blood lipids (total and HDL cholesterol, triglycerides) and fructosamine were determined in EDTA plasma samples stored at $-80{ }^{\circ} \mathrm{C}$ (24). Cholesterol and triglycerides were determined by an enzymatic colorimetric test (Roche Diagnostics). The interassay CV values were $1.7 \%$ for total cholesterol at low and high levels, $6.3 \%$ for HDL cholesterol, $2.2 \%$ for triglycerides at lower levels (1.0 $\mathrm{mmol} / \mathrm{l})$, and $1.8 \%$ at higher levels (1.9 mmol/l). LDL cholesterol was calculated by the following formula (25): $\mathrm{LDL}=$ total cholesterol-HDL cholesterol- $(0.45 \times$ triglycerides).

\section{CVD outcome}

Data on incident non-fatal CVD were based on selfreported events collected through interview conducted in three consecutive cycles: 1998/1999, 2001/2002, and 2005/2006. CVD was identified by three predefined questions about whether the subject had experienced myocardial infarction or other heart diseases, including cardiac arrhythmia, congestive heart failure, and angina pectoris, cerebral stroke, or diseases or abnormalities of the arteries, respectively, since their previous interview. The time of event was defined as the mid-date in the interval between the interview where CVD was first reported and the previous cycle $((26))$. Cardiovascular deaths occurring until 1st January 2007 were identified 
using the cause of death data coded by the Dutch Central Bureau of Statistics (The Netherlands). Cardiovascular deaths were defined by the International Classification of Disease (ICD-10) codes I20-I79 as a primary cause of death (26). The time of event was defined as the date of death.

These outcomes were combined to study the time to first CVD event during follow-up, whether non-fatal or fatal. For censored participants, the end of follow-up was set as the date of death for those deceased before 1st January 2007, or final participation date in a LASA interview for those alive. Maximum observation time was 11.1 years.

\section{Variables collected at baseline}

Weight, height, and waist circumference were measured as described previously $(16,23)$. Participants were asked about smoking and categorized as current, former, or never smokers. They were also asked to present their current medications, which were coded according to the Anatomical Therapeutic Chemical Classification (ATC) system, including hormone replacement therapy (HRT), bisphosphonates, corticosteroids, lipid-lowering medications, anti-hypertensive medications, and anti-diabetic medications. Blood pressure in the arm was measured in the sitting position using a standard mercury sphygmomanometer. As an indicator of socioeconomic status, participants were asked to specify the highest level of education completed, in nine predefined levels ranging from not completed elementary school to university education. This was categorized into years of education ranging from 5 to 18 years. Physical activity was estimated using the validated LASA Physical Activity Questionnaire (LAPAQ) (27), covering minutes spent on household activities, sports, walking outdoors, and bicycling during the previous 2 weeks. The resulting variable was divided into tertiles due to a rightskewed distribution. The presence of aortic calcification was assessed in a subsample of participants living in Amsterdam and surroundings $(n=502)$, with lateral-view conventional $\mathrm{X}$-rays of the thoracic and lumbar spine (T4-L5), according to the EVOS protocol (28).

\section{Statistical analyses}

Statistical analyses were performed using IBM SPSS 20 and $\mathrm{R}$. We performed the Cox proportional hazards regression model to investigate the relationship between plasma OC levels and incident CVD (29). Initial analysis with penalized splines of OC (30) demonstrated a linear relationship with CVD. Plasma OC was therefore treated as a continuous variable. All analyses were adjusted for age and sex.
We considered BMI, smoking, and physical activity as potential confounders. In addition, we examined potentially mediating factors related to insulin resistance and the risk of CVD, including blood pressure, blood lipids, and fructosamine, in a second series of analyses. Waist circumference was initially examined as a measure of abdominal adiposity (31) and a potential mediator between OC and CVD $(11,14,15,16)$, but was replaced by BMI in the resulting model. The two were highly correlated (Spearman's $\rho, 0.87$ in men and 0.83 in women), and BMI was collected in a standardized way across study centers and available for almost all subjects (99.5 vs 95.6\% for waist circumference). Additional analyses were restricted to those not reporting prevalent CVD at baseline, including 832 subjects (63\% of the total sample). Additional analyses were also performed examining non-fatal or fatal CVD respectively. Possible interaction effects of plasma OC with sex and age on CVD were considered by including a three-way interaction term. The significance level, $\alpha$, was set at 0.05 , except for tests of interaction terms where an $\alpha$ value of 0.10 was accepted. After detecting interactions, analyses were performed stratified by sex and age divided at 75 years, corresponding to the median age. Tests and plots of Schoenfeld residuals showed no evidence of timedependent effects of OC, sex, age, or any other included covariates on CVD, indicating that the data met the assumption of non-proportional hazards.

The cross-sectional association between plasma OC and ACSs at baseline was investigated using logistic regression. Based on an observed increased risk of CVD at an ACS of 4 and above in the data, ACSs were dichotomized into ACS $\geq 4$ (indicating calcified lesions of $5 \mathrm{~cm}$ or more) compared with ACS $\leq 3$ (lesions $<5 \mathrm{~cm}$ ). In addition, ACSs were examined as a mediator in the Cox regression model in a complete case analysis $(n=502)$.

\section{Ethical approval}

This study was approved by the Medical Ethics Committee of the VUmc in Amsterdam, The Netherlands, and written informed consent was obtained from all respondents.

\section{Results}

During the median 4.1 (interquartile range 1.4-8.1) years of follow-up, 709 (53.8\%) of the 1319 participants suffered an incident non-fatal or fatal CVD event. Of these, men experienced 366 events (cumulative incidence 56.8\%) and women 343 events (50.8\%). Among the 588 subjects reporting a CVD event during follow-up, 268 reported heart 
disease only, 76 reported cerebral stroke or cerebral hemorrhage only, and 67 reported diseases or abnormalities of the arteries only, while 177 subjects reported at least two of the above-mentioned events. Among the 219 fatal CVD events during follow-up, 151 (69.0\%) were attributed to ischemic heart disease, pulmonary heart disease, and other forms of heart diseases (ICD-10 codes, I20-I52), 53 (24.2\%) were attributed to cerebrovascular diseases (ICD-10 codes, I60-I69), and 15 (6.8\%) were attributed to diseases of arteries, arterioles, and capillaries (ICD-10 codes, I70-I79).

\section{Baseline characteristics}

Mean (range) age at baseline was 75 (65-88) years and 51\% were women. There was a positive relationship between age and plasma OC, and OC concentration was higher in women than in men. Characteristics according to age and sex are presented in Table 1 . When comparing the baseline characteristics of men and women regardless of age, women had a lower smoking prevalence (12 vs $25 \%$ ), a lower educational level (mean 8.1 vs 9.7 years), and a higher BMI (mean $27.6 \mathrm{vs} 26.0 \mathrm{~kg} / \mathrm{m}^{2}$ ), and they reported more time spent on physical activity than men (mean 173 vs $123 \mathrm{~min} /$ day); $P<0.001$ for all comparisons. Blood pressure and plasma lipids also differed between the age and sex strata. The characteristics that varied significantly across the quartiles of plasma OC are shown in Table 2. The proportion of never smokers was higher at higher plasma OC levels, explained by a higher proportion of never smokers among women. There was a trend of decreasing BMI and waist circumference across increasing plasma OC concentrations. As expected, urinary DPD/Cr increased through increasing plasma OC concentration (Table 2). Use of HRT in women was not significantly associated with plasma OC (not shown). Only one subject, having plasma OC in the highest quartile, reported use of bisphosphonates (not shown).

\section{Plasma OC concentration and incident CVD}

We did not observe an overall association between plasma OC and non-fatal or fatal CVD in the Cox regression model: the hazard ratio (HR) was 0.97 (95\% CI 0.90-1.04) per nmol/l higher plasma OC, adjusted for age and sex. However, an interaction term between sex and plasma OC was statistically significant (age-adjusted, $P=0.006$ ). Sex-stratified analyses revealed an inverse relationship between plasma OC and CVD in men, with a reduced risk at a higher plasma OC concentration (Fig. 1). In men, the age-adjusted HR for CVD was 0.88 (0.79-0.99) per nmol/1 higher plasma OC. In women, there was a tendency for a positive association, which was not statistically significant: the age-adjusted HR was 1.06 (95\% CI 0.96-1.16) per nmol/l.

In the overall sample, we observed a statistically significant three-way interaction between plasma OC, sex, and age on the risk of CVD $(P=0.014)$. There was also a statistically significant two-way interaction between plasma OC and sex $(P=0.024)$, while it was less pronounced for plasma OC and age $(P=0.104)$. When stratifying both sexes into two age groups, no association was found between plasma OC and CVD in the younger-old participants (65-74 years) in either sex. However, in the older-old men ( $\geq 75$ years), there was an inverse linear association between plasma OC and CVD, while there was a positive linear association in the older-old women (Fig. 2). Older-old men had a $14 \%$ reduced risk of CVD per $\mathrm{nmol} / \mathrm{l}$ higher plasma OC, while the older-old women had a corresponding 16\% increased risk (Table 3). The associations persisted with adjustment for potential confounders and cardiovascular mediators respectively. When including adjustment for bone turnover (DPD/Cr), the positive relation in the older-old women persisted, while the inverse association in older-old men was slightly attenuated (Table 3).

\section{Additional analyses}

Analyses restricted to those who did not report CVD at baseline yielded similar associations as in the total sample: the HR was 1.01 (95\% CI 0.90-1.13) per nmol/l, with statistically significant interaction terms: $P=0.004$ for a three-way interaction between plasma OC, age, and sex; $P=0.004$ for plasma OC and sex; and $P=0.054$ for plasma OC and age. In older-old women ( $\geq 75$ years), the positive linear association between plasma OC and CVD was even stronger (Fig. 2 and Table 3).

Additional analysis considering non-fatal CVD showed the same patterns but with less precision. The association between plasma OC and non-fatal CVD was statistically significant only in the older-old women, with an age-adjusted HR of 1.16 (95\% CI 1.01-1.33) per $\mathrm{nmol} / \mathrm{l}$ higher plasma OC. The same was found when studying fatal CVD only, with an age-adjusted HR of 1.20 (95\% CI 1.00-1.44) per $\mathrm{nmol} / \mathrm{l}$ for the older-old women, and no statistically significant findings in the other age and sex strata.

Analysis with urinary $\mathrm{DPD} / \mathrm{Cr}$ as exposure revealed a similar relationship with CVD as plasma OC. There was a statistical interaction between sex and urinary DPD/Cr on CVD $(P=0.005)$, but not between age and urinary DPD/Cr. 
Table 1 Baseline characteristics and cardiovascular events during the follow-up in sex and age strata of participants aged 65 years and older, who provided blood samples in the LASA C-cycle 1995/1996 $(n=1319)^{a}$.

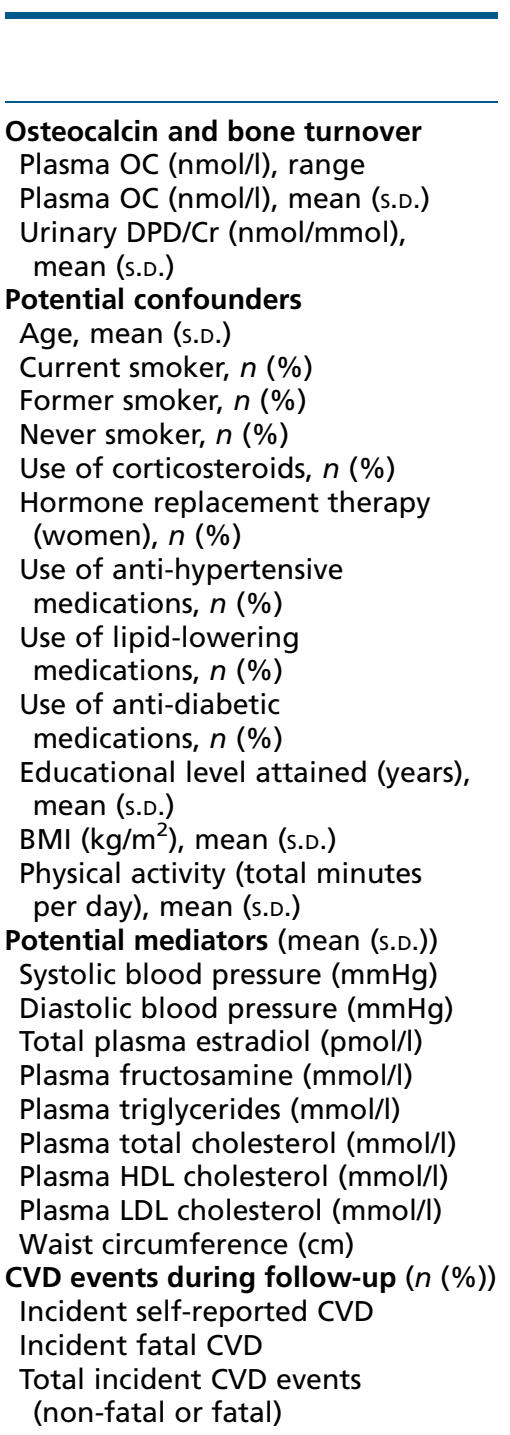

\begin{tabular}{c}
\hline \\
Overall \\
\hline \\
$0.05-9.48$ \\
$2.19(1.11)$ \\
$5.6(2.4)$ \\
\\
$75.6(6.6)$ \\
$241(18.3)$ \\
$609(46.2)$ \\
$469(35.6)$ \\
$106(8.0)$ \\
$13(1.9)$ \\
$528(40.0)$ \\
$64(4.0)$ \\
$76(5.8)$ \\
$8.9(3.3)$ \\
$26.9(4.2)$ \\
$149(97)$ \\
$152.7(26.1)$ \\
$83.2(13.6)$ \\
$54.2(30.9)$ \\
$235(38)$ \\
$1.52(0.78)$ \\
$5.67(1.03)$ \\
$1.33(0.43)$ \\
$3.66(0.95)$ \\
$95.7(11.4)$ \\
$588(44.6)$ \\
$709(53.8)$ \\
\\
\end{tabular}

\begin{tabular}{c}
$\begin{array}{c}\text { Men } 65-74 \\
(n=309)\end{array}$ \\
\hline \\
$0.43-5.85$ \\
$1.82(0.75)$ \\
$4.6(1.9)$
\end{tabular}

\begin{tabular}{c}
$\begin{array}{c}\text { Men 75-88 } \\
(n=335)\end{array}$ \\
\hline \\
$0.37-9.48$ \\
$2.20(1.27)$ \\
$5.4(2.4)$
\end{tabular}

\begin{tabular}{c}
$\begin{array}{c}\text { Women 65-74 } \\
(n=339)\end{array}$ \\
\hline \\
$0.05-8.54$ \\
$2.22(1.04)$ \\
$5.7(2.4)$
\end{tabular}

$69.8(2.7)$
$83(26.9)$
$199(64.4)$
$27(8.7)$
$26(8.4)$
-

$$
\begin{gathered}
81.2(3.8) \\
78(23.3) \\
219(65.4) \\
38(11.3) \\
39(11.6) \\
- \\
131(39.1) \\
8(2.4) \\
21(6.3) \\
9.5(3.5) \\
25.8(3.5) \\
114(88)
\end{gathered}
$$$$
110(35.6)
$$$$
15(4.9)
$$$$
13(4.2)
$$$$
10.0(3.3)
$$$$
26.3(3.3)
$$$$
133(93)
$$

$$
\begin{gathered}
69.9(2.8) \\
61(18.0) \\
116(34.2) \\
162(47.8) \\
21(6.2) \\
5(1.5) \\
111(32.7) \\
31(9.1) \\
17(5.0) \\
8.2(2.9) \\
27.5(4.4) \\
192(99)
\end{gathered}
$$

$151.4(24.2)$
$85.9(14.3)$
$76.3(24.7)$
$229(31)$
$1.57(0.84)$
$5.58(0.95)$
$1.22(0.41)$
$3.65(0.85)$
$99.3(10.1)$

$153(49.5)$
$32(10.4)$
$173(56.0)$
$153.8(26.0)$

\begin{tabular}{|c|c|}
\hline $\begin{array}{c}\text { Women 75-88 } \\
(n=336)\end{array}$ & $P$ value $^{b}$ \\
\hline $0.10-7.87$ & - \\
\hline $2.50(1.16)$ & $<0.001$ \\
\hline $6.7(2.6)$ & $<0.001$ \\
\hline $81.2(3.8)$ & $<0.001$ \\
\hline $19(5.7)$ & \\
\hline 75 (22.3) & \\
\hline $242(72.0)$ & $<0.001$ \\
\hline $20(6.0)$ & 0.024 \\
\hline $8(2.4)$ & 0.39 \\
\hline $176(52.4)$ & $<0.001$ \\
\hline $10(3.0)$ & $<0.001$ \\
\hline $25(7.4)$ & 0.31 \\
\hline $8.0(3.2)$ & $<0.001$ \\
\hline $27.8(5.1)$ & $<0.001$ \\
\hline $153(91)$ & $<0.001$ \\
\hline
\end{tabular}
$81.1(13.5)$
$78.2(25.7)$
$236(40)$
$1.41(0.63)$
$5.28(1.00)$
$1.25(0.40)$
$3.40(0.96)$
$99.0(10.3)$
$150(44.8)$
79 (23.6)
193 (57.6)
$149.3(25.0)$
$83.6(11.9)$
31.4 (13.9)
235 (37)
$1.54(0.85)$
$6.06(0.99)$
$1.47(0.43)$
$3.91(0.96)$
$91.4(11.3)$
$129(38.1)$
31 (9.1)
$148(43.7)$

0.004

$82.5(14.1)<0.001$

$32.9(18.3) \quad<0.001$

$241(42) \quad 0.001$

$1.57(0.78) \quad 0.024$

$5.77(1.03)<0.001$

$1.39(0.42)<0.001$

$3.69(0.96)<0.001$

$93.4(11.6) \quad<0.001$

$156(46.4)$

$77(22.9)$

$195(58.0)$

0.025

$<0.001$

$<0.001$

${ }^{a}$ The number with valid data varies from $n=1319$ (age, sex, plasma OC, smoking, medications, and CVD follow-up) down to $n=1261$ (waist circumference).

${ }^{b}$ One-way ANOVA for comparison of continuous variables or the $\chi^{2}$ test for comparison of percentages across all four strata.

Urinary DPD/Cr was not associated with CVD overall or in men, but positively associated with CVD in women: the age-adjusted HR per $\mathrm{nmol} / \mathrm{mmol}$ of higher urinary $\mathrm{DPD} / \mathrm{Cr}$ was 1.05 (95\% CI 1.01-1.10), and this was not affected by adjustment for plasma OC or by BMI.

\section{Arterial calcification}

No relationship was found between plasma OC and odds of ACS $\geq 4$. In women, we observed reduced estimates at a higher plasma OC concentration, although they did not reach statistical significance: the age-adjusted OR was 0.69 (95\% CI $0.45-1.02$ ) for ACS $\geq 4 / \mathrm{nmol} / \mathrm{l}$ higher plasma OC in older-old women. Additional analysis showed a significantly reduced odds ratio for detectable AC (ACS $\geq 2$ ) compared with no AC (ACS $=1)$ at a higher plasma OC concentration in all women: the OR was 0.76 (95\% CI 0.58-0.99) per nmol/l higher plasma OC in age-adjusted analysis, but no association in men. Including ACSs as a potential mediator in a secondary analysis of the association between plasma OC and CVD did not attenuate the associations (not shown). 
Table 2 Baseline characteristics that varied significantly across quartiles of plasma OC in men and women aged 65 years and older, who provided blood samples in the LASA C-cycle 1995/1996 (all men and women combined).

\begin{tabular}{l}
\hline \\
\hline Osteocalcin and bone turnover \\
Plasma OC (nmol/l), range \\
Plasma OC (nmol/l), mean (s.D.) \\
Urinary DPD/Cr (nmol/mmol), mean (s.D.) \\
Potential confounders \\
Age, mean (s.D.) \\
Sex (women), $n$ (\%) \\
Current smoker, $n(\%)$ \\
Former smoker, $n(\%)$ \\
Never smoker, $n(\%)$ \\
Use of corticosteroids, $n(\%)$ \\
Use of anti-hypertensive \\
medications, $n$ (\%) \\
BMI (kg/m²), mean (s.D.) \\
Potential mediators (mean (s.D.)) \\
Plasma fructosamine (mmol/l) \\
Plasma triglycerides (mmol/l) \\
Plasma HDL cholesterol (mmol/l) \\
Waist circumference (cm) \\
Total plasma estradiol (men; pmol/l) \\
Total plasma estradiol (women; pmol/l)
\end{tabular}

\begin{tabular}{|c|c|}
\hline$n$ & Overall \\
\hline 1319 & $0.05-9.48$ \\
\hline 1319 & $2.19(1.11)$ \\
\hline 1276 & $5.6(2.4)$ \\
\hline 1319 & $75.6(6.6)$ \\
\hline 1319 & 675 (51.2) \\
\hline \multirow[t]{3}{*}{1319} & 241 (18.3) \\
\hline & 609 (46.2) \\
\hline & 469 (35.6) \\
\hline 1319 & $106(8.0)$ \\
\hline 1319 & $528(40.0)$ \\
\hline 1313 & $26.9(4.2)$ \\
\hline 1295 & $235(38)$ \\
\hline 1293 & $1.52(0.78)$ \\
\hline 1292 & $1.33(0.43)$ \\
\hline 1261 & 95.7 (11.4) \\
\hline 635 & $77.3(25.2)$ \\
\hline 664 & $32.1(16.3)$ \\
\hline
\end{tabular}

\begin{tabular}{|c|c|}
\hline Q1 $(n=334)$ & Q2 $(n=329)$ \\
\hline $0.05-1.44$ & $1.45-2.01$ \\
\hline $1.10(0.28)$ & $1.75(0.16)$ \\
\hline $4.8(1.9)$ & $5.1(2.1)$ \\
\hline $74.6(6.4)$ & $74.8(6.4)$ \\
\hline $130(38.9)$ & $144(43.8)$ \\
\hline $60(18.0)$ & $62(18.8)$ \\
\hline $167(50.0)$ & 169 (51.4) \\
\hline $107(32.0)$ & $98(29.8)$ \\
\hline $33(9.9)$ & $36(10.9)$ \\
\hline $161(48.2)$ & $120(36.5)$ \\
\hline $27.9(4.1)$ & $27.1(4.1)$ \\
\hline $240(43)$ & $233(41)$ \\
\hline $1.74(0.94)$ & $1.55(0.76)$ \\
\hline $1.24(0.41)$ & $1.30(0.42)$ \\
\hline $99.6(10.7)$ & $97.2(10.5)$ \\
\hline $82.5(25.7)$ & $75.6(22.9)$ \\
\hline $38.7(24.9)$ & 30.6 (11.7) \\
\hline
\end{tabular}

\begin{tabular}{c}
\hline $\mathbf{Q 3}(n=326)$ \\
\hline \\
$2.02-2.63$ \\
$2.31(0.19)$ \\
$5.6(2.3)$ \\
$75.6(6.5)$ \\
$195(59.8)$ \\
$53(16.3)$ \\
$145(44.5)$ \\
$128(39.3)$ \\
$22(6.7)$ \\
$119(36.5)$ \\
\\
$26.6(4.3)$ \\
\\
$235(35)$ \\
$1.44(0.70)$ \\
$1.39(0.43)$ \\
$93.6(11.4)$ \\
$76.4(24.6)$ \\
$32.1(14.8)$ \\
\hline
\end{tabular}

\begin{tabular}{|c|c|}
\hline Q4 $(n=330)$ & P value ${ }^{a}$ \\
\hline 2.64-9.48 & - \\
\hline 3.61 (1.14) & - \\
\hline $6.9(2.9)$ & $<0.001$ \\
\hline $77.4(6.6)$ & $<0.001$ \\
\hline $206(62.4)$ & $<0.001$ \\
\hline $66(20.0)$ & \\
\hline $128(38.8)$ & \\
\hline $136(41.2)$ & 0.010 \\
\hline $15(4.5)$ & 0.009 \\
\hline $128(38.8)$ & 0.005 \\
\hline $25.8(4.2)$ & $<0.001$ \\
\hline 233 (31) & 0.047 \\
\hline $1.34(0.64)$ & $<0.001$ \\
\hline $1.42(0.43)$ & $<0.001$ \\
\hline $92.2(11.5)$ & $<0.001$ \\
\hline $72.2(26.9)$ & 0.002 \\
\hline $29.0(11.6)$ & $<0.001$ \\
\hline
\end{tabular}

${ }^{a}$ One-way ANOVA for comparison of continuous variables or the $\chi^{2}$ test for comparison of percentages across quartiles.

\section{Discussion}

In this population-based longitudinal cohort study, we found that higher plasma OC concentrations were associated with a reduced risk of CVD in men aged 75 years and older, but with an increased risk of CVD in women aged 75 years and older. We did not find any evidence that associations between plasma OC and CVD were mediated by established metabolic risk factors or by aortic calcification. Plasma OC was unrelated to the risk of CVD in the younger-old subjects.

Despite the growing literature on mechanisms linking OC to the atherosclerotic process, very few studies have investigated the influence of circulating $\mathrm{OC}$ on the risk of CVD in community-dwelling older subjects, and the findings are contradictory. In a cohort of 3542 older men followed for a median of 5.2 years with 198 fatal CVD events, a U-shaped association between plasma OC and fatal CVD was found (20). While CVD mortality was lowest in the second OC quintile, a significant 30\% increased risk was observed in the first quintile and a $69 \%$ increased risk in the fifth quintile. As observed in our study, plasma total $\mathrm{OC}$ at baseline was the exposure measured, and the Cox regression model was adjusted for conventional cardiovascular risk factors. In our study with 111 CVD-related deaths in men and 108 in women, the results suggested a linear positive association between plasma OC and fatal CVD in women only, with an increased risk of CVD at high OC levels, while we could not find any evidence of a non-linear effect. A positive association was also found between serum OC and prevalence of coronary heart disease (CHD) in a crosssectional study of 78 patients with type 2 diabetes mellitus (32). By contrast, inverse associations showing an increased risk of CVD events at low serum OC levels were found in hemodialysis patients (19) and in young survivors of myocardial infarction (18). A cross-sectional study in patients who underwent coronary angiography found a linear negative relationship between serum OC and CHD prevalence (17).

These contradictory results imply that OC measured in plasma may be a correlate of different unmeasured influences among different population subgroups and that causality cannot be inferred at this stage. To what extent OC measured in blood samples reflects OC produced by osteoblasts in bone or in other tissues, such as the arterial walls, is unresolved. Taking cardiovascular risk factors into account did not substantially influence the observed associations between plasma OC and the risk of CVD in our study. However, when taking a marker of bone resorption (urinary DPD/Cr) into account, the association was attenuated and nonsignificant in older-old men, while it was still significant in older-old women. In women, both OC and DPD/Cr 

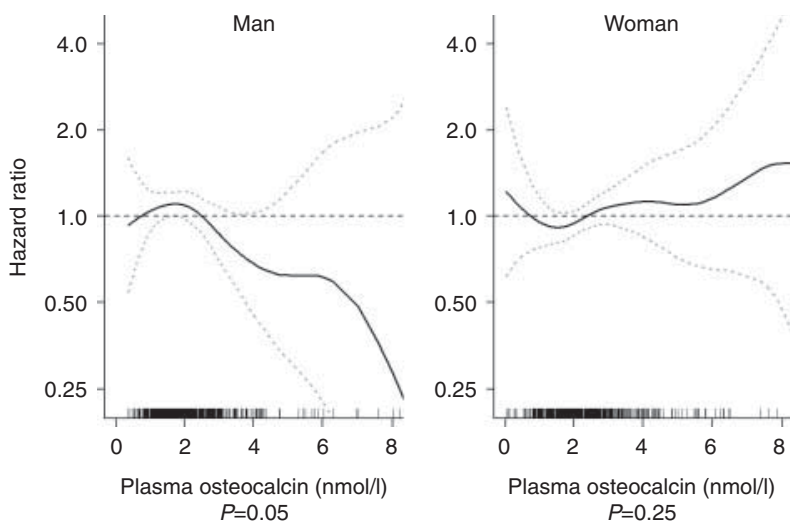

\section{Figure 1}

Age-adjusted hazard ratios with $95 \% \mathrm{Cl}$ of non-fatal or fatal cardiovascular disease across the distribution of plasma $O C$ in men and women. Men: mean (s.D.) plasma OC concentration was 2.01 (1.07) nmol/l, 366 events in 644 participants. Women: mean (s.D.) plasma OC concentration was 2.36 (1.11) nmol/l, 343 events in 675 participants. Rug plots along the horizontal axes represent individual plasma OC concentrations. $P$ values for the linear association between plasma OC and CVD are given.

showed independent positive associations with the risk of CVD. It is recognized that osteoporosis and CVD are age-related conditions that seem to share common characteristics and potentially common underlying pathophysiological mechanisms. Inflammatory cytokines, endogenous sex hormones, lipid oxidation, vitamin $\mathrm{K}$ deficiency, and vitamin $\mathrm{D}$ deficiency have been implicated as possible mediators in the progression of both conditions (33).

The inverse association observed in men is plausible in light of the growing body of evidence from studies in community-dwelling populations $(9,10,11,12,13,14,15)$, including our own study (16), that consistently confirm a relationship between low circulating OC levels and established predictors of CVD. These include central adiposity, hypertension, and unfavorable lipid composition. In the large study in Australian men, where a U-shaped relationship between plasma OC and fatal CVD was found (20), an inverse association between plasma OC and metabolic syndrome was also demonstrated (11). This seemed to be mediated by waist circumference, hyperglycemia, and hypertriglyceridemia. These factors were strongly related to the risk of CVD in men in LASA, but could not provide a link for the negative association between plasma OC and CVD in older-old men in our data. Larger cohorts and inclusion of measurements of undercarboxylated OC are required to clarify the relationship between OC and the risk of CVD in men.

The degree of aortic calcification detectable on lateral radiographs of the spine is strongly predictive of CVD events (34). This was true also for our data. Positive associations between circulating OC and parameters of atherosclerosis were found in a cross-sectional study in 78 participants with type 2 diabetes mellitus (32), in a 6-month follow-up study in 50 participants with type 2 diabetes mellitus (35), and in a study in 67 patients on peritoneal dialysis (36). In the Study of Osteoporotic Fractures, no cross-sectional association was detected between serum OC and abdominal aortic calcification in 363 older women (37). Correspondingly, there was no
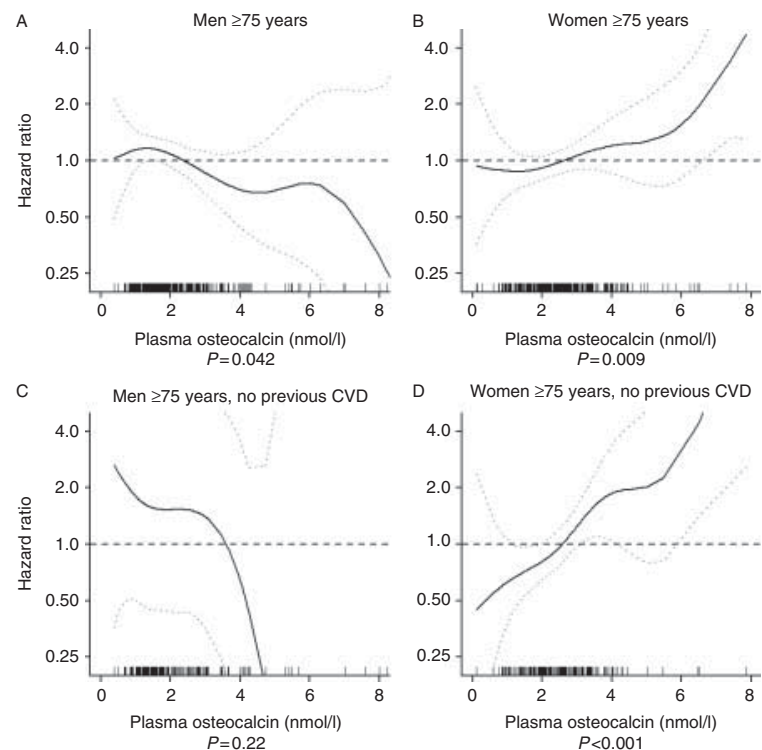

\section{Figure 2}

Age-adjusted hazard ratios with $95 \%$ confidence bands for non-fatal or fatal cardiovascular disease across the distribution of plasma $O C$ in men and women aged 75 years and older.

(A) Men: mean (s.D.) plasma OC concentration was 2.20 (1.27) nmol/l, 193 events in 335 participants. (B) Women: mean (s.D.) plasma OC concentration was 2.50 (1.16) nmol/l, 195 events in 336 participants. (C) Men, restricted to those not reporting CVD at baseline: mean (s.D.) plasma OC concentration was 2.27 (1.43) $\mathrm{nmol} / \mathrm{l}, 57$ events in 174 participants. (D) Women, restricted to those not reporting CVD at baseline: mean (s.D.) plasma OC concentration was $2.45(1.05) \mathrm{nmol} / \mathrm{l}, 82$ events in 200 participants. Rug plots along the horizontal axes represent individual plasma OC concentrations. $P$ values for the linear association between plasma OC and CVD are given. 
Table 3 Sex-and age-specific hazard ratios for incident non-fatal or fatal cardiovascular disease per nmol/l higher plasma OC at baseline.

\begin{tabular}{|c|c|c|c|c|c|c|c|c|c|c|c|}
\hline Sex & $\begin{array}{c}\text { Age at } \\
\text { baseline } \\
\text { (years) }\end{array}$ & $n$ & $\begin{array}{c}\text { CVD } \\
\text { events }\end{array}$ & $\mathbf{H R}^{\mathrm{a}}$ & $95 \% \mathbf{C l}^{\mathrm{a}}$ & $\mathbf{H R}^{\mathrm{b}}$ & $95 \% \mathrm{CI}^{\mathrm{b}}$ & $\mathbf{H R}^{\mathrm{c}}$ & $95 \% \mathrm{Cl}^{\mathrm{C}}$ & $\mathbf{H R}^{\mathrm{d}}$ & $95 \% \mathrm{Cl}^{\mathrm{d}}$ \\
\hline \multicolumn{12}{|c|}{ All participants $(n=1319)$} \\
\hline \multirow[t]{2}{*}{ Men } & $65-74$ & 309 & 173 & 0.95 & $0.77-1.16$ & 1.04 & $0.84-1.31$ & 1.08 & $0.86-1.35$ & 1.15 & $0.91-1.46$ \\
\hline & $75-88$ & 335 & 193 & 0.86 & $0.75-0.99$ & 0.86 & $0.75-1.00$ & 0.84 & $0.72-0.98$ & 0.89 & $0.76-1.03$ \\
\hline \multirow[t]{2}{*}{ Women } & $65-74$ & 339 & 148 & 0.93 & $0.79-1.09$ & 0.96 & $0.82-1.14$ & 0.97 & $0.81-1.16$ & 0.91 & $0.76-1.08$ \\
\hline & $75-88$ & 336 & 195 & 1.16 & $1.03-1.31$ & 1.16 & $1.01-1.32$ & 1.16 & $1.01-1.33$ & 1.15 & $1.00-1.32$ \\
\hline \multicolumn{12}{|c|}{ No previous CVD $(n=832)$} \\
\hline \multirow[t]{2}{*}{ Men } & $65-74$ & 191 & 76 & 1.07 & $0.79-1.45$ & 1.21 & $0.84-1.74$ & 1.26 & $0.86-1.84$ & 1.39 & $0.97-1.98$ \\
\hline & $75-88$ & 174 & 57 & 0.75 & $0.56-1.00$ & 0.71 & $0.52-0.98$ & 0.74 & $0.53-1.01$ & 0.79 & $0.58-1.08$ \\
\hline \multirow[t]{2}{*}{ Women } & $65-74$ & 267 & 84 & 0.90 & $0.73-1.12$ & 0.88 & $0.71-1.10$ & 0.92 & $0.72-1.17$ & 0.86 & $0.67-1.10$ \\
\hline & $75-88$ & 200 & 82 & 1.47 & $1.21-1.79$ & 1.49 & $1.21-1.83$ & 1.49 & $1.17-1.89$ & 1.38 & $1.10-1.73$ \\
\hline
\end{tabular}

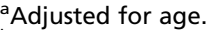

${ }^{b}$ Adjusted for age and potential confounders: BMI, ever smoking, and physical activity.

'Adjusted for age, BMI, and possible cardiovascular mediators: systolic BP, plasma triglycerides, total and HDL cholesterol, and fructosamine.

${ }^{\mathrm{d}}$ Adjusted for age, BMI, and bone resorption (urinary DPD:creatinine ratio).

positive association between plasma OC and ACSs in our data. Thus, our findings did not support a mediating role of aortic calcifications in the relationship between plasma OC and CVD. If anything, there was a suggested inverse association between plasma OC and aortic calcification in older-old women, with a higher degree of aortic calcification at lower plasma OC levels. This corresponds with the results of relatively large studies carried out in community-dwelling populations demonstrating inverse relationships between circulating $\mathrm{OC}$ and progression of atherosclerosis $(17,38,39,40)$. The authors attribute this apparent protective effect of OC to a plausible indirect effect via its regulation of glucose and fat metabolism or other unidentified pathways. One author (39) noted that the effect of OC could be sex specific. Murine data have suggested that $\mathrm{OC}$ regulates testosterone levels in males to modulate fertility, while $O c$-deficient females do not have any fertility abnormality (41).

A high bone turnover measured by urinary $\mathrm{DPD} / \mathrm{Cr}$ predicted CVD, but did not account for the effect of OC in women. Estrogen deficiency causes a dramatic increase in bone resorption after menopause. In LASA, both men and women in the lowest quartile of bioavailable estradiol $\left(\mathrm{E}_{2}\right)$ measured in plasma had a higher bone turnover (plasma OC and urinary $\mathrm{DPD} / \mathrm{Cr}$ ) compared with those in the highest $\mathrm{E}_{2}$ quartile (23). In post-hoc analyses, our observed associations between plasma OC and the risk of CVD could not be attributed to plasma $E_{2}$. By contrast, with adjustment for plasma $\mathrm{E}_{2}$, the positive association between plasma OC and CVD in the older-old women persisted, and the inverse association between plasma OC and
CVD in the oldest men was strengthened (not shown). Moreover, statistical adjustment for plasma testosterone (23) did not influence the association between plasma OC and CVD within the older-old men. Thus, the observed sex differences were not explained by differing sex hormone levels.

Differences in metabolic factors or physical activity did not explain the sex differences (not shown). It may also be speculated whether there may be sex differences in renal function impairment explaining the observed estimates. Men had a higher urinary $\mathrm{Cr}$ concentration than women. However, plasma OC was not associated with urinary $\mathrm{Cr}$ within the sexes, and including urinary $\mathrm{Cr}$ in a post-hoc analysis did not influence the observed associations.

In summary, the reasons for the contradictory findings in men and women could not be identified in this study. We may speculate that the associations are attributable to unidentified factors correlated with circulating OC, such as adiponectin (42) or vitamin K deficiency (33).

A limitation of our study is that information on the proportion of undercarboxylated OC was not available. Therefore, it could not be determined whether risk of CVD was associated with the degree of carboxylation. Experimental studies have suggested that fat and glucose metabolism is regulated by the undercarboxylated isoform (7). Nevertheless, measures of carboxylated or total OC concentrations have been associated with CVD and metabolic risk factors in several studies $(9,11,12,14,20$, $32,40)$, including LASA (16). The use of mid-date between 
interviews due to the lack of exact event dates for selfreported CVD is also a limitation with the potential to dilute the true associations. Another limitation is that only a subsample of 502 participants, all living in Amsterdam and surroundings, had ACS measurements. However, the age and sex distribution and the risk of CVD in the subsample were similar to that in the overall sample, indicating that they were representative of LASA.

\section{Conclusion}

A higher plasma OC concentration was associated with a reduced risk of CVD in older-old men, but with an increased risk in older-old women. The positive association observed in older-old women was more robust. This was not mediated by the established metabolic risk factors for CVD or by aortic calcification. The underlying mechanisms for the observed sex differences represent a challenge for future studies.

\section{Declaration of interest}

The authors declare that there is no conflict of interest that could be perceived as prejudicing the impartiality of the research reported.

\section{Funding}

This study is based on the data collected in the context of the Longitudinal Aging Study Amsterdam (LASA), which is largely funded by the Ministry of Health, Welfare and Sports of The Netherlands. K Holvik was funded by a grant from the Research Council of Norway while writing the manuscript.

\section{Author contribution statement}

$\mathrm{K}$ Holvik prepared the data, performed the statistical analyses and drafted the manuscript, and takes responsibility for the integrity of the data analysis. N M van Schoor, P Lips, and R de Jongh provided scientific advice and contributed to the methods and the design of the manuscript. All coauthors have critically discussed the results, revised the manuscript, and accepted the final version.

\section{Acknowledgements}

The authors would like to thank Jan Poppelaars for his assistance in providing the data.

The work was carried out in the Department of Epidemiology and Biostatistics, EMGO Institute for Health and Care Research, VU University Medical Center, Amsterdam, The Netherlands.

\section{References}

1 Neve A, Corrado A \& Cantatore FP. Osteocalcin: skeletal and extraskeletal effects. Journal of Cellular Physiology 2013228 1149-1153. (doi:10.1002/jcp.24278)
2 Biver E, Chopin F, Coiffier G, Brentano TF, Bouvard B, Garnero P \& Cortet B. Bone turnover markers for osteoporotic status assessment? A systematic review of their diagnosis value at baseline in osteoporosis. Joint, Bone, Spine 201279 20-25. (doi:10.1016/j.jbspin.2011.05.003)

3 Levy RJ, Gundberg C \& Scheinman R. The identification of the vitamin $\mathrm{K}$-dependent bone protein osteocalcin as one of the $\gamma$-carboxyglutamic acid containing proteins present in calcified atherosclerotic plaque and mineralized heart valves. Atherosclerosis 198346 49-56. (doi:10.1016/ 0021-9150(83)90163-6)

4 Idelevich A, Rais Y \& Monsonego-Ornan E. Bone Gla protein increases HIF-1 $\alpha$-dependent glucose metabolism and induces cartilage and vascular calcification. Arteriosclerosis, Thrombosis, and Vascular Biology 201131 e55-e71. (doi:10.1161/ATVBAHA.111.230904)

5 Foresta C, Strapazzon G, De Toni L, Fabris F, Grego F, Gerosa G, Vettore S \& Garolla A. Platelets express and release osteocalcin and co-localize in human calcified atherosclerotic plaques. Journal of Thrombosis and Haemostasis 201311 357-365. (doi:10.1111/ jth.12088)

6 Sullivan TR, Duque G, Keech AC \& Herrmann M. An old friend in a new light: the role of osteocalcin in energy metabolism. Cardiovascular Therapeutics 201331 65-75. (doi:10.1111/j.1755-5922.2011.00300.x)

7 Lee NK, Sowa H, Hinoi E, Ferron M, Ahn JD, Confavreux C, Dacquin R, Mee PJ, McKee MD, Jung DY et al. Endocrine regulation of energy metabolism by the skeleton. Cell 2007 130 456-469. (doi:10.1016/j.cell. 2007.05.047)

8 Ferron M, Hinoi E, Karsenty G \& Ducy P. Osteocalcin differentially regulates $\beta$ cell and adipocyte gene expression and affects the development of metabolic diseases in wild-type mice. PNAS $2008 \mathbf{1 0 5}$ 5266-5270. (doi:10.1073/pnas.0711119105)

9 Chen L, Li Q, Yang Z, Ye Z, Huang Y, He M, Wen J, Wang X, Lu B, Hu J et al. Osteocalcin, glucose metabolism, lipid profile and chronic low-grade inflammation in middle-aged and elderly Chinese. Diabetic Medicine 201330 309-317. (doi:10.1111/j.1464-5491.2012.03769.x)

10 Tan A, Gao Y, Yang X, Zhang H, Qin X, Mo L, Peng T, Xia N \& Mo Z. Low serum osteocalcin level is a potential marker for metabolic syndrome: results from a Chinese male population survey. Metabolism 201160 1186-1192. (doi:10.1016/j.metabol.2011.01.002)

11 Yeap BB, Chubb SA, Flicker L, McCaul KA, Ebeling PR, Beilby JP \& Norman PE. Reduced serum total osteocalcin is associated with metabolic syndrome in older men via waist circumference, hyperglycemia, and triglyceride levels. European Journal of Endocrinology 2010163 265-272. (doi:10.1530/EJE-10-0414)

12 Saleem U, Mosley TH Jr \& Kullo IJ. Serum osteocalcin is associated with measures of insulin resistance, adipokine levels, and the presence of metabolic syndrome. Arteriosclerosis, Thrombosis, and Vascular Biology 201030 1474-1478. (doi:10.1161/ATVBAHA.110.204859)

13 Bae SJ, Choe JW, Chung YE, Kim BJ, Lee SH, Kim HY, Koh JM, Kim HK \& Kim GS. The association between serum osteocalcin levels and metabolic syndrome in Koreans. Osteoporosis International 201122 2837-2846. (doi:10.1007/s00198-010-1504-y)

14 Movahed A, Larijani B, Nabipour I, Kalantarhormozi M, Asadipooya K, Vahdat K, Akbarzadeh S, Farrokhnia M, Assadi M, Amirinejad R et al. Reduced serum osteocalcin concentrations are associated with type 2 diabetes mellitus and the metabolic syndrome components in postmenopausal women: the crosstalk between bone and energy metabolism. Journal of Bone and Mineral Metabolism 201230 683-691. (doi:10.1007/s00774-012-0367-z)

15 Yang R, Ma X, Pan X, Wang F, Luo Y, Gu C, Bao Y \& Jia W. Serum osteocalcin levels in relation to metabolic syndrome in Chinese postmenopausal women. Menopause 201320 548-553. (doi:10.1097/ GME.0b013e31828aa32d)

16 Oosterwerff MM, van Schoor NM, Lips P \& Eekhoff EM. Osteocalcin as a predictor of the metabolic syndrome in older persons: a populationbased study. Clinical Endocrinology 201378 242-247. (doi:10.1111/ j.1365-2265.2012.04391.x) 
17 Zhang Y, Qi L, Gu W, Yan Q, Dai M, Shi J, Zhai Y, Chen Y, Liu J, Wang W et al. Relation of serum osteocalcin level to risk of coronary heart disease in Chinese adults. American Journal of Cardiology 2010106 1461-1465. (doi:10.1016/j.amjcard.2010.07.013)

18 Goliasch G, Blessberger H, Azar D, Heinze G, Wojta J, Bieglmayer C, Wagner $\mathrm{O}$, Schillinger M, Huber K, Maurer G et al. Markers of bone metabolism in premature myocardial infarction ( $\leq 40$ years of age). Bone 201148 622-626. (doi:10.1016/j.bone.2010.11.005)

19 Yamashita T, Okano K, Tsuruta Y, Akiba T \& Nitta K. Serum osteocalcin levels are useful as a predictor of cardiovascular events in maintenance hemodialysis patients. International Urology and Nephrology 201345 207-214. (doi:10.1007/s11255-012-0156-6)

20 Yeap BB, Chubb SA, Flicker L, McCaul KA, Ebeling PR, Hankey GJ, Beilby JP \& Norman PE. Associations of total osteocalcin with all-cause and cardiovascular mortality in older men. The Health In Men Study. Osteoporosis International 201223 599-606. (doi:10.1007/s00198-0111586-1)

21 Huisman M, Poppelaars J, van der Horst M, Beekman AT, Brug J, van Tilburg TG \& Deeg DJ. Cohort profile: the Longitudinal Aging Study Amsterdam. International Journal of Epidemiology 201140 868-876. (doi:10.1093/ije/dyq219)

22 Dumon JC, Wantier H, Mathieu F, Mantia M \& Body JJ. Technical and clinical validation of a new immunoradiometric assay for human osteocalcin. European Journal of Endocrinology 1996135 231-237. (doi:10.1530/eje.0.1350231)

23 Kuchuk NO, van Schoor NM, Pluijm SM, Smit JH, de Ronde W \& Lips P. The association of sex hormone levels with quantitative ultrasound, bone mineral density, bone turnover and osteoporotic fractures in older men and women. Clinical Endocrinology 200767 295-303. (doi:10.1111/j.1365-2265.2007.02882.x)

24 Dik MG, Jonker C, Comijs HC, Deeg DJ, Kok A, Yaffe K \& Penninx BW. Contribution of metabolic syndrome components to cognition in older individuals. Diabetes Care 200730 2655-2660. (doi:10.2337/ dc06-1190)

25 Friedewald WT, Levy RI \& Fredrickson DS. Estimation of the concentration of low-density lipoprotein cholesterol in plasma, without use of the preparative ultracentrifuge. Clinical Chemistry 1972 18 499-502.

26 van Bunderen CC, van Nieuwpoort IC, van Schoor NM, Deeg DJ, Lips P $\&$ Drent ML. The association of serum insulin-like growth factor-I with mortality, cardiovascular disease, and cancer in the elderly: a population-based study. Journal of Clinical Endocrinology and Metabolism 201095 4616-4624. (doi:10.1210/jc.2010-0940)

27 Stel VS, Smit JH, Pluijm SM, Visser M, Deeg DJ \& Lips P. Comparison of the LASA Physical Activity Questionnaire with a 7-day diary and pedometer. Journal of Clinical Epidemiology 200457 252-258. (doi:10.1016/j.jclinepi.2003.07.008)

28 O'Neil TW, Felsenberg D, Varlow J, Cooper C, Kanis JA \& Silman AJ. The prevalence of vertebral deformity in European men and women: the European Vertebral Osteoporosis Study. Journal of Bone and Mineral Research 199611 1010-1018. (doi:10.1002/jbmr.5650110719)

29 Therneau TM. Package 'survival': survival analysis, including penalised likelihood. http://cran.r-project.org/web/packages/survival/survival 4/25/2012, Last access on 15/03/2013.

30 Therneau TM \& Grambsch PM. In Modeling Survival Data. Extending the Cox Model. Springer Science, 2000.
31 Carmienke S, Freitag MH, Pischon T, Schlattmann P, Fankhaenel T, Goebel H \& Gensichen J. General and abdominal obesity parameters and their combination in relation to mortality: a systematic review and meta-regression analysis. European Journal of Clinical Nutrition 201367 573-585. (doi:10.1038/ejcn.2013.61)

32 Reyes-Garcia R, Rozas-Moreno P, Jimenez-Moleon JJ, Villoslada MJ, Garcia-Salcedo JA, Santana-Morales S \& Munoz-Torres M. Relationship between serum levels of osteocalcin and atherosclerotic disease in type 2 diabetes. Diabetes \& Metabolism 201238 76-81. (doi:10.1016/j.diabet. 2011.07.008)

33 Farhat GN \& Cauley JA. The link between osteoporosis and cardiovascular disease. Clinical Cases in Mineral and Bone Metabolism 20085 19-34.

34 Bastos Gonçalves F, Voûte MT, Hoeks SE, Chonchol MB, Boersma EE, Stolker RJ \& Verhagen HJ. Calcification of the abdominal aorta as an independent predictor of cardiovascular events: a meta-analysis. Heart 201298 988-994. (doi:10.1136/heartjnl-2011-301464)

35 Kanazawa I, Yamaguchi T \& Sugimoto T. Relationship between bone biochemical markers versus glucose/lipid metabolism and atherosclerosis; a longitudinal study in type 2 diabetes mellitus. Diabetes Research and Clinical Practice 201192 393-399. (doi:10.1016/j.diabres. 2011.03.015)

36 Janda K, Krzanowski M, Chowaniec E, Kusnierz-Cabala B, Dumnicka P, Krasniak A, Podolec P \& Sulowicz W. Osteoprotegerin as a marker of cardiovascular risk in patients on peritoneal dialysis. Polskie Archiwum Medycyny Wewnetrznej 2013123 149-155.

37 Parker BD, Bauer DC, Ensrud KE \& Ix JH. Association of osteocalcin and abdominal aortic calcification in older women: the study of osteoporotic fractures. Calcified Tissue International 201086 185-191. (doi:10.1007/s00223-010-9332-9)

38 Yang R, Ma X, Dou J, Wang F, Luo Y, Li D, Zhu J, Bao Y \& Jia W. Relationship between serum osteocalcin levels and carotid intimamedia thickness in Chinese postmenopausal women. Menopause 2013 20 1194-1199. (doi:10.1097/GME.0b013e31828aa32d)

39 Confavreux CB, Szulc P, Casey R, Boutroy S, Varennes A, Vilayphiou N, Goudable J \& Chapurlat RD. Higher serum osteocalcin is associated with lower abdominal aortic calcification progression and longer 10-year survival in elderly men of the MINOS cohort. Journal of Clinical Endocrinology and Metabolism 201398 1084-1092. (doi:10.1210/ jc.2012-3426)

40 Kim KJ, Kim KM, Park KH, Choi HS, Rhee Y, Lee YH, Cha BS, Kim MJ, Oh SM, Brown JK et al. Aortic calcification and bone metabolism: the relationship between aortic calcification, BMD, vertebral fracture, 25-hydroxyvitamin D, and osteocalcin. Calcified Tissue International 201291 370-378. (doi:10.1007/s00223-012-9642-1)

41 Oury F, Ferron M, Huizhen W, Confavreux C, Xu L, Lacombe J, Srinivas $\mathrm{P}$, Chamouni A, Lugani F, Lejeune $\mathrm{H}$ et al. Osteocalcin regulates murine and human fertility through a pancreas-bone-testis axis. Journal of Clinical Investigation 2013123 2421-2433. (doi:10.1172/ JCI65952)

42 Luo XH, Zhao LL, Yuan LQ, Wang M, Xie H \& Liao EY. Development of arterial calcification in adiponectin-deficient mice: adiponectin regulates arterial calcification. Journal of Bone and Mineral Research 2009 24 1461-1468. (doi:10.1359/jbmr.090227)
Received 20 December 2013

Revised version received 12 April 2014

Accepted 2 May 2014 